\title{
Uniform existence of the integrated density of states for randomly weighted Hamiltonians on long-range percolation graphs
}

\author{
Slim Ayadi* $\quad$ Fabian Schwarzenberger ${ }^{\dagger} \quad$ Ivan Veselić $^{\dagger}$
}

June 18, 2018

\begin{abstract}
We consider random Hamiltonians defined on long-range percolation graphs over $\mathbb{Z}^{d}$. The Hamiltonian consists of a randomly weighted Laplacian plus a random potential. We prove uniform existence of the integrated density of states and express the IDS using a Pastur-Shubin trace formula.
\end{abstract}

\section{Introduction}

In the study of solution properties and spectral features of random ergodic and periodic operators the integrated density of states (IDS), also known as spectral distribution function, plays and important role. While it is by its very nature a much simpler object than the original one, i.e. the operator family, it exhibits a number of features which turn out to be crucial for the understanding of the spectrum and the corresponding eigensolutions. Let us spell out some of these features explicitly:

(A) the low energy asymptotics of the IDS,

(B) its local and global continuity properties, and

(C) its approximability by finite volume analogues.

In fact, $(\mathrm{C})$ is of relevance for all other questions about the IDS since all methods used to answer/understand them rely on one stage or another on finite volume approximations. Of course, depending on the question which is being considered, the type and quality of the approximation $(\mathrm{C})$ will vary.

Let us highlight the intimate relationship between the continuity (B) and approximability (C) properties on an elementary level, to provide a motivation for the discussion which follows. On the one hand, if a sequence of probability measures converges weakly to a measure with no atoms, the corresponding distribution functions converge already uniformly. On the other hand, uniform convergence of continuous distribution functions obviously implies that the

\footnotetext{
${ }^{*}$ University of Tunis El Manar, Departement of Mathematics, Faculté des Sciences de Tunis, 2092 Tunis, Tunisia

${ }^{\dagger}$ Technische Universität Chemnitz, Fakultät für Mathematik, 09107 Chemnitz, Germany
} 
limiting measure has no atoms. Now, there are important classes of random operators where it is known that the IDS is not continuous (and other, where continuity is still an open question). In this setting the question arises whether the approximability (C) in the $L^{\infty}$ topology persists or not. More precisely, it is natural to ask, what are reasonable criteria, and which is the mechanism, which ensures that the finite volume approximating distribution functions converge uniformly to the IDS.

Let us review the history of works addressing the above explained approximability question. In the simplest setting of finite-hopping range ergodic operators on $\ell^{2}\left(\mathbb{Z}^{d}\right)$ the continuity of the IDS was established already in [10. In fact, this result can be sharpened under very mild conditions to a quantitative form of continuity, namely log-Hölder continuity, c.f. [8].

In the corresponding continuum setting, more specifically for ergodic Schrödinger operators on $L^{2}\left(\mathbb{R}^{d}\right)$, for quite some while the continuity of the IDS was known for specific classes of random potentials, in particular for the alloy type or continuum Anderson potential. This continuity result is typically established via a Wegner estimate, following the exposition in the paper [34]. Actually, this paper treats (discrete) Anderson models on the lattice, but functional analytics tool allow to transfer the methods to the continuum setting. In any case, for this technique a regular distribution of the values of the random potential has to be assumed. For a survey of results in this direction see, e.g. [33] and the references cited there. If no assumption of the regularity of the distribution of the potential values is assumed log-Hölder continuity of the IDS of ergodic Schrödinger operators in one dimension was established in [9]. For dimensions two and three this result was established only very recently in [6]. The case $d \geq 4$ is still open. This is due to the fact that the present state of knowledge about quantitative unique continuation properties of eigensolutions of Schrödinger operators based on Carleman estimates, cf. e.g. [5], is good enough for the IDS-continuity proof in dimensions three and less, but not above this threshold.

Once one moves away from the Euclidean setting, continuity is no longer a prevailing feature of the IDS. There are two important prototypical examples of discrete Laplace and Schrödinger operators whose IDS exhibit jumps: Hamiltonians on percolation clusters [7], [32] and quasi-crystal graphs [20, 21, 16]. In the former model the discontinuities form even a dense set in the spectrum. Nevertheless, it has been established that the sequence of normalized finite volume eigenvalue counting functions converges at every energy and even uniformly along the energy axis to the IDS, cf. [32, 21, 18, 22].

The question whether the approximability $(\mathrm{C})$ holds at any given energy is also of interest in a purely geometric setting, where no randomness in involved. More specifically, the papers [24, 26, 25, 11] study the approximation of Betti numbers by their finite volume analogues. The usual weak convergence of measures is not sufficient to yield this statement. The results of [22, 19, 29] actually apply under very mild and natural geometric assumptions (namely amenability) and thus lift the pointwise everywhere convergence results of [24, 26, 25, 11] to uniform convergence.

However, all papers mentioned so far concern local operators (in the continuum setting) respectively finite-hopping range operators (in the discrete setting). Let us spell out the last property explicitly: A bounded operator $A$ on $\ell^{2}(G)$ for some graph $G$ is called of finite hopping range $R \in \mathbb{N}$ if for any $\varphi \in \ell^{2}(G)$ and $x \in G$ with distance larger than $R$ to $\operatorname{supp} \varphi$

$$
(A \varphi)(x)=0
$$


The first result for an ensemble of operators on $\ell^{2}(G)$ beyond this restriction was achieved in [30]. It studies Laplace operators on long-range percolation graphs. These operators are not of finite hopping range, albeit non-zero "matrix elements far off the diagonal" appear with small probability. Due to the methods applied in [30], the randomness present in the model had to be of finite local complexity.

In the present paper we continue this line of research. Here we are able to treat models with real-valued entries, possibly continuously distributed, and with long-range interactions. As mentioned above, the proof of [30] does not apply in this setting, but a combination of ideas from this paper together with methods from [22] allows one to prove uniform approximability in this more general situation. Also, we will treat here randomized versions of adjacency as well as Laplace operators. The results of [30] apply to the second type of ensembles only.

Question (A) has been addressed for certain long-range percolation models before in [1], extending previous results for the usual quantum percolation model on $\mathbb{Z}^{d}$, cf. for example [15, 27]. We will quote for completeness sake the result of [1] below, once we have the necessary notation at disposal.

Let us stress an important feature of long-range percolation Hamiltonians. They provide a simple model interpolating between discrete random Schrödinger operators and random matrices. This is of interest, since the two last mentioned classes of operators have quite different spectral features. Thus one is led to ask in which aspects and regimes, long-range percolation Hamiltonians share features with one or the other of these classes. To explain the structural difference between discrete random Schrödinger operators and random matrices let us restrict ourselves for the moment to operators on finite segments of $\mathbb{Z}$. The arising Schrödinger operators are a special type of finite Jacobi matrices, and are in particular tridiagonal. Random matrices have a full array of random entries. The non-zero entries of a Laplacian of a typical long-range percolation graph are concentrated mostly near the main diagonal, however no diagonal will consist entirely of zeros. This is the mentioned interpolating property. The relation between long-range percolation models and random matrices was for instance studied in [3, 4].

Let us describe the content of the paper in detail. In the next section we state the main result in a concise form, discuss extensions to groups and graphs beyond $\mathbb{Z}^{d}$ and the relation to results concerning the low energy asymptotics of the IDS (A) established for long-range percolation graphs in [1].

In Section 3 we present the long-range percolation model and in particular the underlying probability space. Furthermore we define the randomly weighted Hamiltonian $H_{\omega}$ on the longrange percolation graph as a selfadjoint and metrically transitive random operator. Depending on the choice of the corresponding parameters, the operator in question is either the adjacency operator of a long-range percolation graph, a Laplacian, or a Schrödinger operator (i.e. a Laplacian plus a random potential), each one with random weights on the edges. For a realization $\omega$ the restriction of this operator to a finite box $\Lambda_{n} \subset \mathbb{Z}^{d}$ gives rise to the eigenvaluecounting function $F_{n}^{\omega}$. This function encodes the distribution of the spectrum of the restricted operator. In the sequel we pursue the question whether and in which sense the limit of the sequence of functions $\left(F_{n}^{\omega}\right)$ exists. In Section 4 , weak convergence of this sequence is established for almost all realizations using a result of Figotin. In Section 5 this statement is upgraded to uniform convergence along the energy axis. This is done by proving that the functions $F_{n}^{\omega}$ do not only approximate the limit function at its continuity points, but also give an efficient estimate of the size of the jumps at a point of discontinuity. The key tool to control the size of the jumps is Theorem 5.5, formulating the main technical contribution of 
the present paper.

Let us stress that we give a detailed account of all the main steps of the proof thus making it accessible to non-specialists.

\section{Main result}

Here we formulate the main result of the paper using a minimum of notation needed for this purpose. More detailed definitions of the framework can be found in Section 3 ,

Denote by $E:=\left\{\{x, y\} \subseteq \mathbb{Z}^{d} \mid x, y \in \mathbb{Z}^{d}\right\}$ the set of all edges (or loops) on $\mathbb{Z}^{d}$ and by $a_{e}, b_{e}, e \in E$ a collection of independent real-valued random variables on a probability space $(\Omega, \mathcal{A}, \mathbb{P})$. The variance of the $a_{e}, e \in E$ is uniformly bounded and any two $a_{\{x, y\}}$ and $a_{\{x+k, y+k\}}$ are identically distributed for $x, y, k \in \mathbb{Z}^{d}$. Let $p \in \ell^{1}\left(\mathbb{Z}^{d}\right)$ with $0 \leq p(x)=p(-x) \leq$ 1 and $b_{\{x, y\}}$ be Bernoulli distributed with parameter $p(x-y)$. The kernel, resp. the matrix entries of the random Hamiltonian $H(\omega)$ are given by:

$$
H_{x, y}(\omega):=H_{x, y}^{\alpha, \beta}(\omega):= \begin{cases}a_{\{x, y\}}(\omega) b_{\{x, y\}}(\omega) & \text { if } x \neq y, \\ \alpha a_{\{x\}}(\omega) b_{\{x\}}(\omega)-\beta \sum_{z \neq x} a_{\{x, z\}}(\omega) b_{\{x, z\}}(\omega) & \text { if } x=y,\end{cases}
$$

where $\alpha, \beta \in[0,1]$ are fixed numbers. Selfadjointness properties of $H(\omega)$ are discussed in Section 3. Depending on the values $\alpha$ and $\beta$ we obtain several interesting subclasses: randomly weighted Laplacians or adjacency operators, with or without random potentials (on the diagonal), cf. Remark 3.4.

For $n \in \mathbb{N}$ let $\Lambda_{n}:=([-n, n] \cap \mathbb{Z})^{d}, H_{n}(\omega)$ be the restriction of $H(\omega)$ to $\Lambda_{n}$, and $F_{n}^{\omega}(\lambda)$ the number of eigenvalues of $H_{n}(\omega)$ not exceeding $\lambda$, counting eigenvalues according to their multiplicity. Set $F: \mathbb{R} \rightarrow \mathbb{R}$,

$$
F(\lambda):=\mathbb{E}\left\{\left\langle E_{H}((-\infty, \lambda]) \delta_{0}, \delta_{0}\right\rangle\right\}
$$

where $\mathbb{E}\{\cdot\}$ is the expectation with respect to the measure $\mathbb{P},\langle\cdot, \cdot\rangle$ is the scalar product in $\ell^{2}(\mathbb{Z})$ and $E_{H(\omega)}((-\infty, \lambda])$ is the spectral projector of $H(\omega)$ on the interval $(-\infty, \lambda]$.

Theorem 2.1. Let $F_{n}^{\omega}, F$ be the distribution functions given above. Then there exists a set $\tilde{\Omega} \subseteq \Omega$ of full measure such that for all $\omega \in \tilde{\Omega}$ we have

$$
\lim _{n \rightarrow \infty} \sup _{\lambda \in \mathbb{R}}\left|\frac{F_{n}^{\omega}}{\left|\Lambda_{n}\right|}(\lambda)-F(\lambda)\right|=0 .
$$

Remark 2.2. The limit of the functions $F_{n}^{\omega} /\left|\Lambda_{n}\right|$ for $n \rightarrow \infty$ is called the integrated density of states (IDS). Theorem 2.1 shows that this limit exists in the topology of uniform convergence. Moreover, the theorem provides the equality of the IDS with the function $F$ given in (2.2), which is the expectation of an diagonal element of associated spectral projector. Note that by translation invariance we obtain for any finite $\Lambda \subseteq \mathbb{Z}^{d}$

$$
F(\lambda)=\mathbb{E}\left\{\left\langle E_{H}((-\infty, \lambda]) \delta_{0}, \delta_{0}\right\rangle\right\}=\frac{1}{|\Lambda|} \mathbb{E}\left\{\sum_{x \in \Lambda}\left\langle E_{H}((-\infty, \lambda]) \delta_{x}, \delta_{x}\right\rangle\right\}
$$

Thus the IDS, originally obtained as a macroscopic limit, can be identified as an averged trace per unit volume. An equality of this type is called Pastur-Shubin trace formula. 


\section{Extension to more general geometries}

In the presentation of our results we have not striven for the maximal possible generality, but rather tried to present proofs as explicitly as possible in their most accessible form. This concerns in particular the restriction to operators defined on $\ell^{2}\left(\mathbb{Z}^{d}\right)$. In fact, the presented results carry over to operators on $\ell^{2}(\Gamma)$, where $\Gamma$ may be a much more general graph than the lattice $\mathbb{Z}^{d}$. The explicit calculations in this case can be found in the submitted Thesis [31. Here we will only state only the scope of the general results:

Let $G$ be a finitely generated discrete amenable group, and $\Gamma$ a graph on which $G$ acts freely and cocompactly by translations. Completely analogously as in the $\mathbb{Z}^{d}$ setting one can define long-range percolation on such graphs, as well as random operators introduced in Definition (2.1), respectively (3.5) . For such models the results which we use from [28] can be proven analogously. Consequently, Lemma 3.6 and Theorem 4.2 have their generalized counterparts in this setting. Relying on the ideas of [22] and [30] one can see that it is possible to extend the results of Section 5, as well. Here one needs to apply the pointwise ergodic theorem of Lindenstrauss 23 instead of Theorem 5.1 to finally obtain that Theorem 2.1 holds analogously.

Let us note that the method we use here is in the sense efficient, that it does not need any condition beyond amenability, i.e. the existence of a Følner sequence, on the discrete group $G$. In comparison to this, the method of [30] relies on the following additional tiling condition:

It is assumed that there exists a Følner sequence $\left(Q_{n}\right)_{n}$ such that for each $n \in \mathbb{N}$ there is a set $T_{n}=T_{n}^{-1} \subseteq G$ with the property that $G$ is the disjoint union of the sets $\left\{Q_{n} t \mid t \in T_{n}\right\}$. This assumption is satisfied for many amenable groups, however it is not clear whether it holds for all of them.

\section{Low energy asymptotics}

In [1] the authors study low energy asymptotics of the IDS for the long-range percolation model. Again, let $p \in \ell^{1}\left(\mathbb{Z}^{d}\right)$ with $0 \leq p(x)=p(-x) \leq 1$ and $b_{\{x, y\}}$ be Bernoulli distributed with parameter $p(x-y)$. We define for each $\omega$ the set $E_{\omega}:=\left\{\{x, y\} \subseteq \mathbb{Z}^{d} \mid b_{\{x, y\}}(\omega)=1\right\}$. The operator under consideration is the (non-weighted) Laplacian $\Delta_{\omega}$ of the graph $\left(\mathbb{Z}^{d}, E_{\omega}\right)$, i.e. the operator acting on any $\varphi: \mathbb{Z}^{d} \rightarrow \mathbb{C}$ with finite support by

$$
\left(\Delta_{\omega} \varphi\right)(x)=\sum_{\substack{y \neq x \\\{x, y\} \in E_{\omega}}}(\varphi(x)-\varphi(y)) .
$$

This equals the operator in (2.1) in the case $\alpha=0, \beta=1$ and where the random variables $a_{e}$ are constant 1 , see also (3.6)

An in physical communities common way to introduce the long-range percolation model is the following: For each pair of vertices $x, y \in \mathbb{Z}^{d}$ let $J_{x, y}$ be a non-negative real number such that

- $J_{x, y}=J_{y, x}$

- $J_{x+z, y+z}=J_{x, y}$ for all $z \in \mathbb{Z}^{d}$,

- $J:=J_{x}:=\sum_{y \in \mathbb{Z}^{d}} J_{x, y}$ is finite and independent of $x \in \mathbb{Z}^{d}$. 
We fix $\beta>0$ and declare an edge $\{x, y\}$ to be an element of $E_{\omega}$ with probability $1-\mathrm{e}^{-\beta J_{x, y}}$. This gives the random graph $\Gamma_{\omega}=\left(\mathbb{Z}^{d}, E_{\omega}\right)$. Notice that the probability that certain edge is an element of $E_{\omega}$ is increasing in $\beta$. Thus, the subcritical phase, in which all clusters are almost surely finite corresponds to small values of the parameter $\beta$ and the supercritical phase in which there exists almost surely an infinite cluster corresponds to large values of the parameter $\beta$. Just like in the case of the nearest neighbor percolation model these two phases are separated by a single value of the parameter $\beta$. The authors of [1] define the IDS as in (2.2) and prove that for every subcritical $\beta$ there are constants $c(\beta), d(\beta)>0$ such that for $E>0$ small enough

$$
\exp \left(-c(\beta) E^{-1 / 2}\right) \leq F(E)-F(0) \leq \exp \left(-d(\beta) E^{-1 / 2}\right) .
$$

Actually the results of [1] apply to operators on quasi-transitive graphs $\Gamma$. The present result is complementary to these observations, as we show that the finite volume approximants do actually converge to this limit function given by the Pastur-Shubin formula. Furthermore the combination of both results shows that even the approximating functions will exhibit exponential behavior for low energies.

Note that in this paper we introduce the long-range percolation model in another, but equivalent (see [30]) way, via a certain function $p \in \ell^{1}\left(\mathbb{Z}^{d}\right)$, see (3.1). More background on the models considered in [1] can be found in the review paper [2].

\section{$3 \quad$ Setting and first results}

Let $\Gamma$ be the $\mathbb{Z}^{d}$ lattice and denote by $d: \mathbb{Z}^{d} \times \mathbb{Z}^{d} \rightarrow \mathbb{N}_{0}$ the graph distance in the lattice or equivalently the $\ell^{1}$-distance in $\mathbb{Z}^{d}$. With this metric we define the $R$-boundary of a set $\Lambda \subseteq \mathbb{Z}^{d}$ by

$$
\partial^{R} \Lambda:=\left\{x \in \Lambda \mid d(x, y) \leq R \text { for some } y \in \mathbb{Z}^{d} \backslash \Lambda\right\} .
$$

Furthermore we let $E:=\left\{\{x, y\} \subseteq \mathbb{Z}^{d} \mid x, y \in \mathbb{Z}^{d}\right\}$ be the set of all subsets of $\mathbb{Z}^{d}$ containing either one or two elements. The set $E$ can be interpreted as the edge set of the complete undirected graph over $\mathbb{Z}^{d}$, containing loops at each vertex.

The probability space $(\Omega, \mathcal{A}, \mathbb{P})$ is given in the following way. The sample space is $\Omega=$ $\prod_{e \in E}(\mathbb{R} \times\{0,1\})$ and we denote the elements in $\Omega$ by $\omega=\left(\omega_{e}^{\prime}, \omega_{e}^{\prime \prime}\right)_{e \in E}$. The appropriate $\sigma$-algebra is $\mathcal{A}=\bigotimes_{e \in E}(\mathcal{B}(\mathbb{R}) \otimes \mathcal{P}(\{0,1\}))$. In order to define a measure on this space we fix some $p \in \ell^{1}\left(\mathbb{Z}^{d}\right)$ with

$$
0 \leq p(x)=p(-x) \leq 1 \quad\left(x \in \mathbb{Z}^{d}\right)
$$

and for each $z \in \mathbb{Z}^{d}$ some probability measure $\mu_{z}$ on $\mathbb{R}$ such that there is $v \in \mathbb{R}$ with

$$
\int_{\mathbb{R}} x^{2} d \mu_{z}(x) \leq v^{2} \quad\left(z \in \mathbb{Z}^{d}\right) .
$$

We set $\mathbb{P}:=\bigotimes_{\{x, y\} \in E}\left(\mu_{x-y} \otimes \nu_{x-y}\right)$ where for each $z \in \mathbb{Z}^{d}$ the measure $\nu_{z}$ is Bernoulli with parameter $p(z)$.

Remark 3.1. The $\sigma$-algebra $\mathcal{A}$ is generated by the cylinder sets $\mathcal{Z}$, which are given the following way

$$
\mathcal{Z}=\left\{Z\left(A_{e_{1}}, B_{e_{1}}, \ldots, A_{e_{k}}, B_{e_{k}}\right) \mid k \in \mathbb{N}, e_{i} \in E, A_{e_{i}} \in \mathcal{B}(\mathbb{R}), B_{e_{i}} \in \mathcal{P}(\{0,1\}) \text { for } i=1, \ldots, k\right\}
$$


where

$$
Z\left(A_{e_{1}}, B_{e_{1}}, \ldots, A_{e_{k}}, B_{e_{k}}\right)=\left\{\omega \in \Omega \mid \omega_{e_{i}}^{\prime} \in A_{e_{i}}, \omega_{e_{i}}^{\prime \prime} \in B_{e_{i}} \text { for } i=1, \ldots, k\right\} .
$$

Now for each $\omega=\left(\omega_{e}^{\prime}, \omega_{e}^{\prime \prime}\right)_{e \in E}$ and $e \in E$ we set $a_{e}(\omega):=\omega_{e}^{\prime}$ and $b_{e}(\omega):=\omega_{e}^{\prime \prime}$. This procedure gives independent random variables $a_{e}, b_{e}, e \in E$ satisfying $\mathbb{P}\left(a_{e} \in B\right)=\mu_{e}(B)$ as well as $\mathbb{P}\left(b_{e}=1\right)=\nu_{e}(\{1\})=p(x-y)$ for arbitrary $e=\{x, y\} \in E$ and $B \in \mathcal{B}(\mathbb{R})$. Furthermore, by (3.2) we have for each $e \in E$

$$
\mathbb{E}\left(\left|a_{e}\right|\right) \leq v^{2}+1
$$

These random variables induce for each $\omega \in \Omega$ a graph $\Gamma_{\omega}=\left(\mathbb{Z}^{d}, E_{\omega}\right)$ with weighted edges. Here $\mathbb{Z}^{d}$ is the vertex set and $E_{\omega}$ is the subset of $E$, where an edge $e \in E$ is an element of $E_{\omega}$ if and only if $b_{e}(\omega)=1$. In this case one can think of $a_{e}(\omega)$ as the weight of the edge $e$. For a subset $\Lambda \subseteq \mathbb{Z}^{d}$ and an element $x \in \mathbb{Z}^{d}$ we write $x \stackrel{\omega}{\sim} \Lambda$ if there exists $y \in \Lambda$ with $\{x, y\} \in E_{\omega}$.

The following Lemma shows that $\Gamma_{\omega}$ is almost surely locally finite, i.e. with probability one each vertex is incident to only finitely many edges in $\Gamma_{\omega}$.

Lemma 3.2. The graph $\Gamma_{\omega}$ is locally finite for almost all $\omega \in \Omega$.

Proof. Fix an element $x \in \mathbb{Z}^{d}$ and consider the events $A_{y}:=\left\{b_{\{x, y\}}=1\right\}, y \in \mathbb{Z}^{d}$. Then clearly

$$
\sum_{y \in \mathbb{Z}^{d}} \mathbb{P}\left(A_{y}\right)=\sum_{y \in \mathbb{Z}^{d}} p(x-y)<\infty,
$$

as $p \in \ell^{1}\left(\mathbb{Z}^{d}\right)$. Hence, the Borel-Cantelli Lemma gives a set $\Omega_{x}$ of full measure such that each $\omega \in \Omega_{x}$ is contained in only finitely many $A_{y}, y \in \mathbb{Z}^{d}$. As $\mathbb{Z}^{d}$ is countable $\tilde{\Omega}:=\bigcap_{x \in \mathbb{Z}^{d}} \Omega_{x}$ is a set of full measure as well. Furthermore $\Gamma_{\omega}$ is locally finite for all $\omega \in \tilde{\Omega}$.

Given $\gamma \in \mathbb{Z}^{d}$, let us define translations $T_{\gamma}: \Omega \rightarrow \Omega$ by

$$
T_{\gamma}(\omega)=T_{\gamma}\left(\left(\omega_{e}^{\prime}, \omega_{e}^{\prime \prime}\right)_{e \in E}\right)=\left(\omega_{e+\gamma}^{\prime}, \omega_{e+\gamma}^{\prime \prime}\right)_{e \in E}
$$

where for $e=\{g, h\} \in E$ we mean by $e+\gamma$ the element $\{g+\gamma, h+\gamma\} \in E$. For $\gamma \in \mathbb{Z}^{d}$ and $B \in \mathcal{A}$ we denote the image and the preimage of $B$ under $T_{\gamma}$ by

$$
T_{\gamma}(B)=\left\{T_{\gamma}(\omega) \in \Omega \mid \omega \in B\right\} \quad \text { and } \quad T_{\gamma}^{-1}(B)=\left\{\omega \in \Omega \mid T_{\gamma}(\omega) \in B\right\} .
$$

Note that for $B \in \mathcal{A}$ we have $T_{\gamma}^{-1}(B)=T_{\gamma^{-1}}(B)$. We further define $T$ to be the mapping $\gamma \mapsto T_{\gamma}$ which maps each element of $\mathbb{Z}^{d}$ into the space of automorphisms on $(\Omega, \mathcal{A}, \mathbb{P})$. Note that by definition $T$ is ergodic if and only if for any $B \in \mathcal{A}$ with $T_{\gamma}(B)=B$ for all $\gamma \in \mathbb{Z}^{d}$ one has $\mathbb{P}(B) \in\{0,1\}$. The following result is basic, but we do not know an explicit reference in the literature, so we include a proof for completeness sake.

Lemma 3.3. $T$ is a measure preserving, ergodic left-action on $(\Omega, \mathcal{A}, \mathbb{P})$.

Proof. For an edge $e=\{g, h\} \in E$, vertices $x, y \in \mathbb{Z}^{d}$ and $\omega \in \Omega$ we have $T_{0}(\omega)=\omega$ and

$$
T_{x+y}(\omega)=\left(\omega_{e+x+y}^{\prime}, \omega_{e+x+y}^{\prime \prime}\right)_{e \in E}=T_{x}\left(T_{y}(\omega)\right)
$$

which shows that $T$ is a left action of $\mathbb{Z}^{d}$ on $\Omega$. 
By definition of $\mathbb{P}$ and the random variables $a_{e}$ and $b_{e}$ we have $\mathbb{P}\left(a_{e} \in B\right)=\mathbb{P}\left(a_{e+\gamma} \in B\right)$ as well as $\mathbb{P}\left(b_{e}=1\right)=\mathbb{P}\left(b_{e+\gamma}=1\right)$ for any $e \in E, \gamma \in \mathbb{Z}^{d}$ and $B \in \mathcal{B}(\mathbb{R})$. Furthermore, as $T_{\gamma}$ is a translation, $\mathbb{P}(Z)=\mathbb{P}\left(T_{\gamma}(Z)\right)$ holds obviously for any $\gamma \in \mathbb{Z}^{d}$ and any cylinder set $Z \in \mathcal{Z}$, which implies the same property for any set $B \in \mathcal{A}$, c.f. Remark 3.1.

To prove ergodicity let $B \in \mathcal{A}$ with $B=T_{\gamma}(B)$ for all $\gamma \in \mathbb{Z}^{d}$ and $\mathbb{P}(B)>0$ be given. We need to show that this implies $\mathbb{P}(B)=1$. In the following we apply the approximation lemma for measures, which belongs to the entourage of Carathéodory's extension theorem, cf. e.g. Theorem 1.65 in [17]. Let $\varepsilon>0$. As $B \in \mathcal{A}=\sigma(\mathcal{Z})$ and as $\mathcal{Z}$ is a semiring we can find cylinder sets $Z_{1}, \ldots, Z_{n} \in \mathcal{Z}$ such that

$$
\mathbb{P}(B \triangle Z)<\varepsilon \quad \text { where } \quad Z:=\bigcup_{k=1}^{n} Z_{k},
$$

which gives

$$
\mathbb{P}(B)^{2}-2 \mathbb{P}(B) \varepsilon \leq \mathbb{P}(Z)^{2} \leq \mathbb{P}(B)^{2}+2 \mathbb{P}(B) \varepsilon+\varepsilon^{2} .
$$

Furthermore we have for any $\gamma \in \mathbb{Z}^{d}$

$$
\begin{aligned}
\mathbb{P}\left(Z \cap T_{\gamma} Z\right) & \leq \mathbb{P}\left((B \cup(Z \backslash B)) \cap T_{\gamma} Z\right) \\
& \leq \mathbb{P}\left(B \cap T_{\gamma} Z\right)+\mathbb{P}\left((Z \backslash B) \cap T_{\gamma} Z\right) \\
& \leq \mathbb{P}\left(B \cap\left(T_{\gamma} B \cup\left(T_{\gamma} Z \backslash T_{\gamma} B\right)\right)\right)+\varepsilon \\
& \leq \mathbb{P}\left(B \cap T_{\gamma} B\right)+\mathbb{P}\left(B \cap\left(T_{\gamma} Z \backslash T_{\gamma} B\right)\right)+\varepsilon \\
& \leq \mathbb{P}\left(B \cap T_{\gamma} B\right)+2 \varepsilon
\end{aligned}
$$

By symmetry we get for all $\gamma \in \mathbb{Z}^{d}$

$$
\mathbb{P}\left(B \cap T_{\gamma} B\right)-2 \varepsilon \leq \mathbb{P}\left(Z \cap T_{\gamma} Z\right) \leq \mathbb{P}\left(B \cap T_{\gamma} B\right)+2 \varepsilon
$$

and the $T$-invariance of $B$ implies

$$
\mathbb{P}(B)-2 \varepsilon \leq \mathbb{P}\left(Z \cap T_{\gamma} Z\right) \leq \mathbb{P}(B)+2 \varepsilon .
$$

As $Z$ is a finite union of cylinder sets, it does only depend on finitely many edges. Hence there exists an element $h \in \mathbb{Z}^{d}$ such that $Z$ and $T_{h} Z$ are independent, which gives

$$
\mathbb{P}\left(Z \cap T_{h} Z\right)=\mathbb{P}(Z) \mathbb{P}\left(T_{h} Z\right)=\mathbb{P}(Z)^{2}
$$

since $T$ is measure preserving. This gives together with (3.3) and (3.4)

$$
\mathbb{P}(B)-2 \mathbb{P}(B) \varepsilon-\varepsilon^{2}-2 \varepsilon \leq \mathbb{P}(B)^{2} \leq \mathbb{P}(B)+2 \mathbb{P}(B) \varepsilon+2 \varepsilon
$$

and dividing by $\mathbb{P}(B)>0$ leads to

$$
1-2 \varepsilon-\frac{\varepsilon^{2}+2 \varepsilon}{\mathbb{P}(B)} \leq \mathbb{P}(B) \leq 1+2 \varepsilon+\frac{2 \varepsilon}{\mathbb{P}(B)}
$$

As these inequalities hold for arbitrary $\mathbb{P}(B) \geq \varepsilon>0$ we get $\mathbb{P}(B)=1$. 
Denote by $\ell^{2}\left(\mathbb{Z}^{d}\right)$ all square summable, complex-valued functions on $\mathbb{Z}^{d}$ and by $C_{c}\left(\mathbb{Z}^{d}\right)$ the subset of $\ell^{2}\left(\mathbb{Z}^{d}\right)$ consisting of all finitely supported functions. Let $\alpha, \beta \in[0,1]$ be some fixed numbers. Using the random variables $a_{e}, b_{e}, e \in E$ we define for each $\omega \in \tilde{\Omega}$ as in Lemma 3.2 the random operator $\tilde{H}(\omega):=\tilde{H}^{\alpha, \beta}(\omega): C_{c}\left(\mathbb{Z}^{d}\right) \rightarrow \ell^{2}\left(\mathbb{Z}^{d}\right)$ point-wise by

$$
\tilde{H}_{x, y}(\omega):=\tilde{H}_{x, y}^{\alpha, \beta}(\omega):= \begin{cases}a_{\{x, y\}}(\omega) b_{\{x, y\}}(\omega) & \text { if } x \neq y, \\ \alpha a_{\{x\}}(\omega) b_{\{x\}}(\omega)-\beta \sum_{z \neq x} a_{\{x, z\}}(\omega) b_{\{x, z\}}(\omega) & \text { if } x=y\end{cases}
$$

and for $\varphi \in C_{c}\left(\mathbb{Z}^{d}\right)$ we set

$$
(\tilde{H}(\omega) \varphi)(x):=\left(\tilde{H}^{\alpha, \beta}(\omega) \varphi\right)(x):=\sum_{y \in \mathbb{Z}^{d}} \tilde{H}_{x, y}(\omega) \varphi(y) .
$$

It is easy to see that

$$
(\tilde{H}(\omega) \varphi)(x)=\sum_{\substack{y \neq x \\\{x, y\} \in E_{\omega}}}(\varphi(y)-\beta \varphi(x)) a_{\{x, y\}}(\omega)+\alpha \varphi(x) a_{\{x\}}(\omega) .
$$

Using this we obtain for each $\varphi \in C_{c}\left(\mathbb{Z}^{2}\right)$ and $\omega \in \Omega$ such that $\Gamma_{\omega}$ is locally finite that $\tilde{H}(\omega) \varphi \in \ell^{1}\left(\mathbb{Z}^{d}\right) \subseteq \ell^{2}\left(\mathbb{Z}^{d}\right)$. To see this we set $A:=\operatorname{supp} \varphi, m:=\max _{x \in A}|\varphi(x)|$ and $N_{y}(\omega):=$ $\left\{x \in \mathbb{Z}^{d} \mid\{x, y\} \in E_{\omega}\right\}$ to estimate

$$
\begin{aligned}
\sum_{x \in \mathbb{Z}^{d}}\left|\sum_{y \in \mathbb{Z}^{d}} \tilde{H}_{x, y}(\omega) \varphi(y)\right| & \leq \sum_{x \in \mathbb{Z}^{d}} \sum_{y \in A}\left|\tilde{H}_{x, y}(\omega)\right||\varphi(y)| \leq m \sum_{x \in \mathbb{Z}^{d}} \sum_{y \in A}\left|\tilde{H}_{x, y}(\omega)\right| \\
& \leq m \sum_{y \in A} \sum_{x \in N_{y}(\omega)}\left|\tilde{H}_{x, y}(\omega)\right| \leq m \sum_{y \in A}\left(\left|\tilde{H}_{y, y}(\omega)\right|+\sum_{\substack{x \in N_{y}(\omega) \\
x \neq y}}\left|\tilde{H}_{x, y}(\omega)\right|\right) \\
& \leq m \sum_{y \in A}\left(\left|a_{\{y\}}(\omega)\right|+2 \sum_{\substack{x \in N_{y}(\omega) \\
x \neq y}}\left|\tilde{H}_{x, y}(\omega)\right|\right)<\infty .
\end{aligned}
$$

Note that here we used that $N_{y}(\omega)$ is finite, as the underlying graph $\Gamma_{\omega}$ is locally finite. In the sense of [28, Section §.1.B] the mapping

$$
\tilde{H}: \Omega \rightarrow L\left(\ell^{2}\left(\mathbb{Z}^{d}\right)\right), \quad \omega \mapsto \begin{cases}\tilde{H}(\omega) & \text { if } \omega \in \tilde{\Omega}, \\ \operatorname{Id} & \text { else. }\end{cases}
$$

is a random operator with domain $C_{c}\left(\mathbb{Z}^{d}\right)$. This means that almost surely $C_{c}\left(\mathbb{Z}^{d}\right)$ is a subset of the domain of $\tilde{H}$ and almost surely $\tilde{H} u$ is for all $u \in C_{c}\left(\mathbb{Z}^{d}\right)$ a random vector. Note that here $L\left(\ell^{2}\left(\mathbb{Z}^{d}\right)\right)$ is the space of the linear operators which are densely defined in $\ell^{2}\left(\mathbb{Z}^{d}\right)$.

Remark 3.4. The operator $\tilde{H}(\omega)$ depends on the choice of $\alpha, \beta \in[0,1]$ and is defined on the finitely supported functions in $\ell^{2}\left(\mathbb{Z}^{d}\right)$. In Lemma 3.6 we will define the self-adjoint extension $H(\omega)$ of this operator. Depending on $\alpha$ and $\beta$ we have in particular the following special cases for $H(\omega)$ :

- if $\alpha=0$ and $\beta=1$, then $H(\omega)$ is the randomly weighted Laplacian on the graph $\Gamma_{\omega}$, 
- if $\alpha=\beta=1$, then $H(\omega)$ is the randomly weighted Laplacian on the graph $\Gamma_{\omega}$ plus a random diagonal,

- if $\alpha=1$ and $\beta=0$, then $H(\omega)$ is the randomly weighted adjacency operator of $\Gamma_{\omega}$ with a random diagonal,

- if $\alpha=\beta=0$, then $H(\omega)$ is the randomly weighted adjacency operator of $\Gamma_{\omega}$ with zeros on the diagonal.

The diagonal elements which appear if $\alpha>0$ can be interpreted, either as random weights on the loops or as a random potential. For values $\alpha, \beta \in(0,1)$ the operator can be seen as an interpolation between, the adjacency operator and the Laplacian respectively Schrödinger operator of the graph $\Gamma_{\omega}$.

We will use the same symbol $T_{\gamma}$ for a mapping $T_{\gamma}: L\left(\ell^{2}\left(\mathbb{Z}^{d}\right)\right) \rightarrow L\left(\ell^{2}\left(\mathbb{Z}^{d}\right)\right)$ defined by

$$
T_{\gamma}\left(\left(A_{x, y}\right)_{x, y \in \mathbb{Z}^{d}}\right):=\left(A_{x+\gamma, y+\gamma}\right)_{x, y \in \mathbb{Z}^{d}},
$$

for arbitrary $A=\left(A_{x, y}\right)_{x, y \in \mathbb{Z}^{d}} \in L\left(\ell^{2}\left(\mathbb{Z}^{d}\right)\right)$. We set $U_{\gamma}: \ell^{2}\left(\mathbb{Z}^{d}\right) \rightarrow \ell^{2}\left(\mathbb{Z}^{d}\right)$

$$
U_{\gamma}\left((\varphi(x))_{x \in \mathbb{Z}^{d}}\right):=(\varphi(x+\gamma))_{x \in \mathbb{Z}^{d}}
$$

where $\varphi=(\varphi(x))_{x \in \mathbb{Z}^{d}}$ is arbitrary. Then obviously $T_{\gamma}(A)=U_{\gamma} A U_{\gamma}^{-1}$.

For each $x, y, \gamma \in \mathbb{Z}^{d}$ with $x \neq y$ and $\omega=\left(\omega_{e}^{\prime}, \omega_{e}^{\prime \prime}\right)_{e \in E}$ we set $s:=\{x, y\}$ and have

$$
\begin{aligned}
\tilde{H}_{x, y}\left(T_{\gamma}(\omega)\right) & =a_{s}\left(T_{\gamma}(\omega)\right) b_{s}\left(T_{\gamma}(\omega)\right) \\
& =a_{s}\left(\left(\omega_{e+\gamma}^{\prime}, \omega_{e+\gamma}^{\prime \prime}\right)_{e \in E}\right) b_{s}\left(\left(\omega_{e+\gamma}^{\prime}, \omega_{e+\gamma}^{\prime \prime}\right)_{e \in E}\right) \\
& =\omega_{s+\gamma}^{\prime} \cdot \omega_{s+\gamma}^{\prime \prime} \\
& =\omega_{\{x+\gamma, y+\gamma\}}^{\prime} \cdot \omega_{\{x+\gamma, y+\gamma\}}^{\prime \prime} \\
& =a_{\{x+\gamma, y+\gamma\}}(\omega) \cdot b_{\{x+\gamma, y+\gamma\}}(\omega)=\tilde{H}_{x+\gamma, y+\gamma}(\omega)
\end{aligned}
$$

Furthermore we have for the diagonal elements

$$
\begin{aligned}
\tilde{H}_{x, x}\left(T_{\gamma}(\omega)\right) & =\alpha a_{\{x\}}\left(T_{\gamma}(\omega)\right) b_{\{x\}}\left(T_{\gamma}(\omega)\right)-\beta \sum_{z \neq x} a_{\{x, z\}}\left(T_{\gamma}(\omega)\right) b_{\{x, z\}}\left(T_{\gamma}(\omega)\right) \\
& =\alpha a_{\{x+\gamma\}}(\omega) b_{\{x+\gamma\}}(\omega)-\beta \sum_{z \neq x} a_{\{x+\gamma, z+\gamma\}}(\omega) b_{\{x+\gamma, z+\gamma\}}(\omega)=\tilde{H}_{x+\gamma, x+\gamma}(\omega) .
\end{aligned}
$$

Therefore we have

$$
\tilde{H}\left(T_{\gamma}(\omega)\right)=T_{\gamma}(\tilde{H}(\omega)) .
$$

Definition 3.5. Let $A$ be a random operator mapping each element of the probability space $(\Omega, \mathcal{A}, \mathbb{P})$ to an linear operator on the Hilbert space $\mathcal{H}$. Then $A$ is called metrically transitive, if there exists a group $\mathcal{T}$ of measure preserving automorphisms of $(\Omega, \mathcal{A}, \mathbb{P})$, a group of unitary operators $\mathcal{U}:=\left\{U_{T} \mid T \in \mathcal{T}\right\}$ on $\mathcal{H}$ and a homomorphism from $\mathcal{T}$ to $\mathcal{U}$ such that

$$
B \in \mathcal{A} \text { such that } T B=B \text { for all } T \in \mathcal{T} \quad \Rightarrow \quad \mathbb{P}(B) \in\{0,1\}
$$

and one has for all $\omega \in \Omega$ and all $T \in \mathcal{T}$ the relation

$$
A(T \omega)=U_{T} A(\omega) U_{T}^{-1} .
$$


The next aim is to prove that $\tilde{H}$ is essentially selfadjoint and that $\tilde{H}$ and its selfadjoint extension are metrically transitive.

Lemma 3.6. Let $(\Omega, \mathcal{A}, \mathbb{P})$ and the random operator $\tilde{H}$ be given as above. Then

(a) there exists a set $\Omega^{\prime}$ of full measure such that for each $\omega \in \Omega^{\prime}$ the operator $\tilde{H}(\omega)$ is essentially self-adjoint. We denote the closure of $\tilde{H}(\omega)$ by $H_{\omega}$ and its domain by $D(\omega)$.

(b) the random operators $\tilde{H}$ and

$$
H: \Omega \rightarrow L\left(\ell^{2}\left(\mathbb{Z}^{d}\right)\right) \text { given by } H(\omega)= \begin{cases}H_{\omega} & \text { if } \omega \in \Omega^{\prime} \\ \text { Id } & \text { otherwise }\end{cases}
$$

are metrically transitive. Here $\mathrm{Id}$ is the identity operator in $L\left(\ell^{2}\left(\mathbb{Z}^{d}\right)\right)$.

To prove the Lemma we will make use of the following theorem due to Figotin [12], see also [28].

Theorem $3.7([12],[28])$. Let $(\Omega, \mathcal{A}, \mathbb{P})$ be a probability space and $A$ a metrically transitive random operator with domain $C_{c}\left(\mathbb{Z}^{d}\right)$ satisfying

$$
\mathbb{E}\left(\left(\sum_{x \in \mathbb{Z}^{d}}\left|A_{0, x}\right|\right)^{2}\right)<\infty
$$

Then the operator $A(\omega)$ is for almost all $\omega \in \Omega$ essentially self-adjoint.

Proof of Lemma 3.6. First we show that $\tilde{H}$ is metrically transitive. To this end, define $\mathcal{T}$ and $\mathcal{U}$ as follows

$$
\mathcal{T}:=\left\{T_{\gamma} \mid \gamma \in \mathbb{Z}^{d}\right\}, \quad \mathcal{U}:=\left\{U_{T_{\gamma}}:=U_{\gamma} \mid \gamma \in \mathbb{Z}^{d}\right\} .
$$

and set $\varphi: \mathcal{T} \rightarrow \mathcal{U}, \varphi\left(T_{\gamma}\right)=U_{\gamma}$, which clearly is a homomorphism. It is obvious that $\mathcal{T}$ and $\mathcal{U}$ are groups and it is easy to prove that each $U_{\gamma}$ is unitary. Furthermore we know from Lemma 3.3 that the translations $T_{\gamma}$ are measure preserving automorphisms of the probability space. Property (3.8) follows from the ergodicity of $T$ shown in Lemma 3.3 as well. From line (3.7) we infer that (3.9) holds.

In order to apply Theorem 3.7. to show that $\tilde{H}$ is almost surely essentially selfadjoint, it remains to prove (3.10) for $\tilde{H}$. Therefore we consider for each $\omega \in \Omega$

$$
\begin{aligned}
\left(\sum_{x \in \mathbb{Z}^{d}}\left|\tilde{H}_{0, x}(\omega)\right|\right)^{2} & =\left(\left|H_{0,0}(\omega)\right|+\sum_{x \neq 0}\left|H_{0, x}(\omega)\right|\right)^{2} \\
& \leq\left(\alpha\left|a_{\{0\}}(\omega) b_{\{0\}}(\omega)\right|+(\beta+1) \sum_{x \neq 0}\left|a_{\{0, x\}}(\omega) b_{\{0, x\}}(\omega)\right|\right)^{2} \\
& \leq 4\left(\sum_{x \in \mathbb{Z}^{d}}\left|a_{\{0, x\}}(\omega)\right| b_{\{0, x\}}(\omega)\right)^{2} .
\end{aligned}
$$

For each $\omega \in \Omega$ set $N(\omega):=\left\{x \in \mathbb{Z}^{d} \mid b_{\{0, x\}}(\omega)=1\right\}$. By Lemma 3.2 there exists a set $\tilde{\Omega} \subseteq \Omega$ of full measure such that $|N(\omega)|<\infty$ for all $\omega \in \tilde{\Omega}$. For $\omega \in \tilde{\Omega}$ we have

$$
\left(\sum_{x \in \mathbb{Z}^{d}}\left|a_{\{0, x\}}(\omega)\right| b_{\{0, x\}}(\omega)\right)^{2}=\left(\sum_{x \in N(\omega)}\left|a_{\{0, x\}}(\omega)\right|\right)^{2} \leq|N(\omega)| \cdot \sum_{x \in N(\omega)}\left|a_{\{0, x\}}(\omega)\right|^{2} .
$$


Taking the expectation value on both sides and the application of the monotone convergence theorem leads to

$$
\mathbb{E}\left(\left(\sum_{x \in \mathbb{Z}^{d}}\left|a_{\{0, x\}}(\omega)\right| b_{\{0, x\}}(\omega)\right)^{2}\right) \leq \mathbb{E}\left(\left(\sum_{x \in \mathbb{Z}^{d}}\left|a_{\{0, x\}}\right|^{2} b_{\{0, x\}}|N|\right)\right) \leq v^{2} \sum_{x \in \mathbb{Z}^{d}} \mathbb{E}\left(b_{\{0, x\}}|N|\right),
$$

where $v^{2}$ is the upper bound for the second moments given in (3.2). For each $x \in \mathbb{Z}^{d}$ and $\omega \in \Omega$ we set $N_{x}(\omega):=|N(\omega) \backslash\{x\}|$, then we obtain for fixed $x \in \mathbb{Z}^{d}$

$$
\mathbb{E}\left(b_{\{0, x\}}|N|\right)=\sum_{k=1}^{\infty} k \cdot \mathbb{P}\left(b_{\{0, x\}}(\omega)=1, N_{x}(\omega)=k-1\right)=\mathbb{P}\left(b_{\{0, x\}}(\omega)=1\right) \mathbb{E}\left(N_{x}+1\right) .
$$

Using $\mathbb{E}\left(N_{x}\right) \leq \mathbb{E}(|N|)=\|p\|_{1}<\infty$ this implies

$$
\mathbb{E}\left(\left(\sum_{x \in \mathbb{Z}^{d}}\left|a_{\{0, x\}}(\omega)\right| b_{\{0, x\}}(\omega)\right)^{2}\right) \leq v^{2}(\mathbb{E}(|N|)+1) \sum_{x \in \mathbb{Z}^{d}} p(x)=v^{2}\left(\|p\|_{1}^{2}+\|p\|_{1}\right)<\infty .
$$

This shows together with (3.11) the finiteness of the expression in (3.10) for the operator $\tilde{H}$. Hence Theorem 3.7 gives a set $\Omega^{\prime}$ of full measure such that for each $\omega \in \Omega^{\prime}$ the operator $\tilde{H}(\omega)$ is essentially selfadjoint. This proves of part (a).

To complete the prove of part (b) it remains to show that the operator $H$ is metrically transitive. This follows by the same argument as we used to prove metrically transitivity of $\tilde{H}$. Note that here we use that (3.7) hold for $H$ as well.

The operator $H$ defined as in Lemma 3.6 is a random operator with domain $C_{c}\left(\mathbb{Z}^{d}\right)$, c.f. [28]. We will refer to this operator as weighted Hamiltonian on the graph $\Gamma_{\omega}$.

Let $\left(\Lambda_{n}\right)$ be a sequence of cubes given by

$$
\Lambda_{n}:=([-n, n] \cap \mathbb{Z})^{d} \quad(n \in \mathbb{N})
$$

and for each $n \in \mathbb{N}$ let $H_{n}(\omega)$ be the restriction of $H(\omega)$ to $\Lambda_{n}$. To be precise, for $\Lambda \subseteq \mathbb{Z}^{d}$ let the inclusion $i_{\Lambda}: \ell^{2}(\Lambda) \rightarrow \ell^{2}\left(\mathbb{Z}^{d}\right)$ and the projection $p_{\Lambda}: \ell^{2}\left(\mathbb{Z}^{d}\right) \rightarrow \ell^{2}(\Lambda)$ be given by

$$
\left(i_{\Lambda} \varphi\right)(x)=\left\{\begin{array}{ll}
\varphi(x) & \text { if } x \in \Lambda \\
0 & \text { otherwise }
\end{array} \text { and } \quad\left(p_{\Lambda} \psi\right)(y)=\psi(y)\right.
$$

for all $x \in \mathbb{Z}^{d}, y \in \Lambda, \varphi \in \ell^{2}(\Lambda)$ and $\psi \in \ell^{2}\left(\mathbb{Z}^{d}\right)$. Then we set

$$
H_{n}(\omega):=p_{\Lambda_{n}} H(\omega) i_{\Lambda_{n}}: \ell^{2}\left(\Lambda_{n}\right) \rightarrow \ell^{2}\left(\Lambda_{n}\right)
$$

For each $\omega \in \Omega$ and $n \in \mathbb{N}$ we define a function $F_{n}^{\omega}: \mathbb{R} \rightarrow \mathbb{R}$ by

$$
F_{n}^{\omega}(\lambda):=\mid\left\{\text { eigenvalues of } H_{n}(\omega) \text { not exceeding } \lambda\right\} \mid,
$$

where we count the eigenvalues with their multiplicity. Therefore $F_{n}^{\omega}$ is the distribution function of a measure which we will denote by $\rho_{n}^{(\omega)}$. Note that $\left|\Lambda_{n}\right|^{-1} \rho_{n}^{(\omega)}$ is a probability measure. 


\section{Weak convergence}

In order to prove weak convergence of the approximating distribution functions we make use of an abstract result by Figotin [12], see also Theorem 4.8 in [28], which we now cite in a special case.

Theorem $4.1([12],[28])$. Let $(\Omega, \mathcal{A}, \mathbb{P})$ be a probability space and $A$ a metrically transitive random operator with domain $C_{c}\left(\mathbb{Z}^{d}\right)$ such that

$$
\sum_{x \in \mathbb{Z}^{d}} \mathbb{E}\left(\left|A_{0, x}\right|\right)<\infty
$$

and assume that $A$ is almost surely self-adjoint. Then there exists a set $\tilde{\Omega} \subseteq \Omega$ of full measure such that for all $\omega \in \tilde{\Omega}$ and all $\lambda \in\{s \in \mathbb{R} \mid F$ is continuous in $s\}$ one has

$$
\lim _{n \rightarrow \infty} \frac{F_{n}^{\omega}(\lambda)}{\left|\Lambda_{n}\right|}=F(\lambda)
$$

where the limit $F: \mathbb{R} \rightarrow[0,1]$ given by $\lambda \mapsto \mathbb{E}\left\{\left\langle E_{A}((-\infty, \lambda]) \delta_{0}, \delta_{0}\right\rangle\right\}$ is a distribution function of a probability measure. Note that here $E_{A(\omega)}((-\infty, \lambda])$ is the spectral projection in the interval $(-\infty, \lambda]$ of the operator $A(\omega)$ and $\delta_{x} \in \ell^{2}\left(\mathbb{Z}^{d}\right)$ denotes the element with $\delta_{x}(y)=1$ if $x=y$ and $\delta_{x}(y)=0$ otherwise.

This theorem and the previous considerations immediately give the following theorem.

Theorem 4.2. Let the probability space $(\Omega, \mathcal{A}, \mathbb{P})$ and the operator $H$ be the weighted Hamiltonian given in Section 2. Set

$$
F: \mathbb{R} \rightarrow[0,1], \quad F(\lambda):=\mathbb{E}\left\{\left\langle E_{H}((-\infty, \lambda]) \delta_{0}, \delta_{0}\right\rangle\right\} .
$$

Then there exists a set $\tilde{\Omega} \subseteq \Omega$ of full measure, such that for all $\omega \in \tilde{\Omega}$ the distribution functions $F_{n}^{\omega} /\left|\Lambda_{n}\right|$ converge to the distribution function $F$ point-wise at all points of continuity of $F$.

Proof. By definition the operator $H(\omega)$ is self-adjoint for all $\omega \in \Omega$. Furthermore $H$ has domain $C_{c}\left(\mathbb{Z}^{d}\right)$ and $H$ is metrically transitive by Lemma 3.6. The finiteness of $\sum_{x \in \mathbb{Z}^{d}} \mathbb{E}\left(\left|H_{0, x}\right|\right)$ follows from

$$
\begin{aligned}
\sum_{x \in \mathbb{Z}^{d}} \mathbb{E}\left(\left|H_{0, x}\right|\right) & \leq \alpha \mathbb{E}\left(\left|a_{\{0\}}\right| b_{\{0\}}\right)+\beta \mathbb{E}\left(\sum_{x \neq 0}\left|a_{\{0, x\}}\right| b_{\{0, x\}}\right)+\sum_{x \neq 0} \mathbb{E}\left(\left|a_{\{0, x\}}\right| b_{\{0, x\}}\right) \\
& \leq 2 \sum_{x \in \mathbb{Z}^{d}} \mathbb{E}\left(\left|a_{\{0, x\}}\right| b_{\{0, x\}}\right) \leq 2\left(v^{2}+1\right)\|p\|_{1}<\infty
\end{aligned}
$$

where $v^{2}$ is the upper bound for the second moments given in (3.2). Hence, Theorem 4.1 implies the claim of the theorem.

\section{Control of the jumps}

The aim of this section is to control the jumps of the limit function given in Corollary 4.2 in order to obtain uniform convergence of the approximants. In the following we will make use of Birkhoff's ergodic theorem in the $d$-dimensional case, see [13]. 
Theorem 5.1. Let $\mathbb{Z}^{d}$ act from the left on a probability space $(\Omega, \mathcal{A}, \mathbb{P})$ by an ergodic and measure preserving transformation $T$ an let $\left(\Lambda_{n}\right)$ be the sequence of cubes given as in (3.12). Then for any $f \in L^{1}(\mathbb{P})$

$$
\lim _{n \rightarrow \infty} \frac{1}{\left|\Lambda_{n}\right|} \sum_{g \in \Lambda_{n}} f\left(T_{g} \omega\right)=\int f(\omega) d \mathbb{P}(\omega)
$$

holds almost surely.

Lemma 5.2. Let $(\Omega, \mathcal{A}, \mathbb{P})$ be the probability space, $H$ the randomly weighted Hamiltonian and $\left(\Lambda_{n}\right)$ be the sequence of cubes given as in (3.12). Then there exists a set $\tilde{\Omega} \subseteq \Omega$ of full measure such that for all $\omega \in \tilde{\Omega}$ and all $\lambda \in \mathbb{R}$ we have

$$
\lim _{n \rightarrow \infty} \frac{\operatorname{Tr}\left(\chi_{\Lambda_{n}} E_{H(\omega)}(\{\lambda\})\right)}{\left|\Lambda_{n}\right|}=\mathbb{E}\left\{\left\langle E_{H(\omega)}(\{\lambda\}) \delta_{0}, \delta_{0}\right\rangle\right\} .
$$

Proof. Let $\omega \in \Omega$ be fixed. By definition of the trace we have

$$
\operatorname{Tr}\left(\chi_{\Lambda_{n}} E_{H(\omega)}(\{\lambda\})\right)=\sum_{x \in \mathbb{Z}^{d}}\left\langle\chi_{\Lambda_{n}} E_{H(\omega)}(\{\lambda\}) \delta_{x}, \delta_{x}\right\rangle=\sum_{x \in \Lambda_{n}}\left\langle E_{H(\omega)}(\{\lambda\}) \delta_{x}, \delta_{x}\right\rangle .
$$

Let $\operatorname{Eig}(H(\omega), \lambda)$ denote the eigenspace of $H(\omega)$ corresponding to the value $\lambda$, which could possibly be empty if $\lambda$ is not an eigenvalue. Given $\gamma \in \mathbb{Z}^{d}$, we have $\varphi \in \operatorname{Eig}(H(\omega), \lambda)$ if and only if $T_{\gamma}(\varphi) \in \operatorname{Eig}\left(T_{\gamma}(H(\omega)), \lambda\right)$.

Using this we prove

$$
\left\langle E_{T_{z}(H(\omega))}(\{\lambda\}) \delta_{0}, \delta_{0}\right\rangle=\left\langle E_{H(\omega)}(\{\lambda\}) \delta_{z}, \delta_{z}\right\rangle .
$$

Therefore let $\delta_{0}^{\prime} \in \operatorname{Eig}\left(T_{z}(H(\omega)), \lambda\right)$ and $\delta_{0}^{\prime \prime} \in \operatorname{Eig}\left(T_{z}(H(\omega)), \lambda\right)^{\perp}$ such that $\delta_{0}=\delta_{0}^{\prime}+\delta_{0}^{\prime \prime}$. Then we obtain

$$
\left\langle E_{T_{z}(H(\omega))}(\{\lambda\}) \delta_{0}, \delta_{0}\right\rangle=\left\langle E_{T_{z}(H(\omega))}(\{\lambda\}) \delta_{0}^{\prime}, \delta_{0}\right\rangle+\left\langle E_{T_{z}(H(\omega))}(\{\lambda\}) \delta_{0}^{\prime \prime}, \delta_{0}\right\rangle=\left\langle\delta_{0}^{\prime}, \delta_{0}\right\rangle
$$

and with the above equivalence we get

$$
\begin{aligned}
\left\langle\delta_{0}^{\prime}, \delta_{0}\right\rangle & =\left\langle T_{-z}\left(\delta_{0}^{\prime}\right), T_{-z}\left(\delta_{0}\right)\right\rangle \\
& =\left\langle E_{H(\omega)}(\{\lambda\}) T_{-z}\left(\delta_{0}^{\prime}\right), T_{-z}\left(\delta_{0}\right)\right\rangle+\left\langle E_{H(\omega)}(\{\lambda\}) T_{-z}\left(\delta_{0}^{\prime \prime}\right), T_{-z}\left(\delta_{0}\right)\right\rangle \\
& =\left\langle E_{H(\omega)}(\{\lambda\}) \delta_{z}, \delta_{z}\right\rangle,
\end{aligned}
$$

which implies (5.2). Applying (5.1), (5.2) and the fact $T_{x}(H(\omega))=H\left(T_{x}(\omega)\right)$ leads to

$$
\frac{\operatorname{Tr}\left(\chi_{\Lambda_{n}} E_{H(\omega)}(\{\lambda\})\right)}{\left|\Lambda_{n}\right|}=\frac{1}{\left|\Lambda_{n}\right|} \sum_{x \in \Lambda_{n}}\left\langle E_{H\left(T_{x}(\omega)\right)}(\{\lambda\}) \delta_{0}, \delta_{0}\right\rangle .
$$

Finally we use Lemma 3.3 and Theorem 5.1 to obtain the existence of a set $\tilde{\Omega} \subseteq \Omega$ of measure one such that for each $\omega \in \tilde{\Omega}$ we have

$$
\lim _{n \rightarrow \infty} \frac{\operatorname{Tr}\left(\chi_{\Lambda_{n}} E_{H(\omega)}(\{\lambda\})\right)}{\left|\Lambda_{n}\right|}=\int_{\Omega}\left\langle E_{H(\omega)}(\{\lambda\}) \delta_{0}, \delta_{0}\right\rangle d \mathbb{P}(\omega)
$$

which was to prove. 
The following fact is taken from [22]

Lemma 5.3. Let $r>0, \Lambda \subseteq \mathbb{Z}^{d}$ and $U \subseteq \ell^{2}(\Lambda)$ be given and denote by $U_{r}$ the subspace of $U$ consisting of all functions which vanish on $\partial^{r}(\Lambda)$. Then

$$
0 \leq \operatorname{dim}(U)-\operatorname{dim}\left(U_{r}\right) \leq\left|\partial^{r}(\Lambda)\right| .
$$

Proof. Let $P: U \rightarrow \ell^{2}\left(\partial^{r}(\Lambda)\right)$ be the natural projection with $(P \varphi)(x)=\varphi(x)$ for all $x \in$ $\partial^{r}(\Lambda)$. Then we have

$$
0 \leq \operatorname{dim}(U)-\operatorname{dim}(\operatorname{ker} P)=\operatorname{dim}(\operatorname{ran} P) \leq\left|\partial^{r}(\Lambda)\right|,
$$

which proves the claim as $\operatorname{ker} P=U_{r}$.

For given $\omega \in \Omega, R \in \mathbb{N}$ and $Q \subseteq \mathbb{Z}^{d}$ finite, let $L^{(\omega)}(R, Q)$ denote the number of $e \in E$ with $b_{e}(\omega)=1$ which are of length not less than $R$ and incident to some vertex in $Q$, i.e.

$$
L^{(\omega)}(R, Q):=\mid\left\{\{x, y\} \in E \mid b_{\{x, y\}}(\omega)=1, d(x, y) \geq R \text { and }\{x, y\} \cap Q \neq \emptyset\right\} \mid .
$$

Let $\left(\Lambda_{n}\right)$ be the sequence of cubes given as in (3.12). We chose a function $R: \mathbb{N} \rightarrow \mathbb{N}$ such that

$$
\lim _{n \rightarrow \infty} R(n)=\infty \quad \text { and } \quad \lim _{n \rightarrow \infty} \frac{\left|\partial^{R(n)} \Lambda_{n}\right|}{\left|\Lambda_{n}\right|}=0
$$

and set

$$
L_{n}^{(\omega)}:=L^{(\omega)}\left(R(n), \Lambda_{n}\right)
$$

Beside this we set for $R \geq 0$

$$
\varepsilon_{R}:=\sum_{x \in \mathbb{Z}^{d}, d(0, x) \geq R} p(x)
$$

and for $n \in \mathbb{N}_{0}$

$$
\varepsilon(n):=\varepsilon_{R(n)} \quad \text { as well as } \quad \delta(n):=(2 n+1)^{-d / 4} .
$$

Note as $p \in \ell^{1}\left(\mathbb{Z}^{d}\right)$ we have by the definition of $R(n)$ that

$$
\lim _{n \rightarrow \infty} \varepsilon(n)=\lim _{n \rightarrow \infty} \delta(n)=0 .
$$

The next result estimates the probability that the number of long edges is large.

Lemma 5.4. Let $(\Omega, \mathcal{A}, \mathbb{P})$ be given as above. Then the following holds:

(a) There exist constants $R_{0} \in \mathbb{N}$ and $\bar{\delta}>0$ such that for all $0<\delta<\bar{\delta}$, all $R \geq R_{0}$ and all finite $Q \subseteq \mathbb{Z}^{d}$

$$
\mathbb{P}\left(L^{(\omega)}(R, Q) \geq|Q|\left(\varepsilon_{R}+\delta\right)\right) \leq \exp \left(-\frac{\delta^{2}|Q|}{4}\right) .
$$

(b) Let $R: \mathbb{N} \rightarrow \mathbb{N}$ be as in (5.4) and $L_{n}^{(\omega)}=L^{(\omega)}\left(R(n), \Lambda_{n}\right)$. Then there exists a set $\tilde{\Omega} \subseteq \Omega$ of full measure such that for each $\omega \in \tilde{\Omega}$ there exists $n_{0}(\omega)$ with

$$
L_{n}^{(\omega)} \leq\left|\Lambda_{n}\right|(\varepsilon(n)+\delta(n)) \quad\left(n \geq n_{0}(\omega)\right) .
$$


Proof. The proof of part (a) is to be found in [30]. It is basically an application of a Bernstein inequality. Let us prove part (b). Therefore consider the events

$$
A_{n}:=\left\{\omega \in \Omega\left|L_{n}^{(\omega)} \geq\right| \Lambda_{n} \mid(\varepsilon(n)+\delta(n))\right\} .
$$

Then part (a) shows that for $n$ large enough we have

$$
\mathbb{P}\left(A_{n}\right) \leq \exp \left(-\delta(n)^{2}\left|\Lambda_{n}\right| / 4\right)=\exp \left(-(2 n+1)^{d / 2} / 4\right),
$$

which clearly gives $\sum_{n \in \mathbb{N}} \mathbb{P}\left(A_{n}\right)<\infty$. By the Lemma of Borel Cantelli we have

$$
\mathbb{P}\left(\limsup _{n \rightarrow \infty} A_{n}\right)=0
$$

which implies the claim of part (b).

We use Lemmas 5.2, 5.3 and 5.4 to obtain a result similar to Lemma 6.2 in [22]

Theorem 5.5. Let $(\Omega, \mathcal{A}, \mathbb{P})$ be the probability space, $H$ the randomly weighted Hamiltonian, $\left(\Lambda_{n}\right)$ be the sequence of cubes and $\rho_{n}^{(\omega)}$ the measures associated to the eigenvalue counting functions given as before. Then there exists a set $\tilde{\Omega} \subseteq \Omega$ of full measure such that for all $\omega \in \tilde{\Omega}$ and all $\lambda \in \mathbb{R}$ we have

$$
\lim _{n \rightarrow \infty} \frac{\rho_{n}^{(\omega)}(\{\lambda\})}{\left|\Lambda_{n}\right|}=\mathbb{E}\left\{\left\langle E_{H}(\{\lambda\}) \delta_{0}, \delta_{0}\right\rangle\right\}
$$

Proof. Let $\tilde{\Omega} \subseteq \Omega$ be a set of full measure such that the results of Lemma 5.2 and of Lemma 5.4 (b) hold for all $\omega \in \tilde{\Omega}$. We fix some $\omega \in \tilde{\Omega}$ and $\lambda \in \mathbb{R}$. With the function $R: \mathbb{N} \rightarrow \mathbb{N}$ given in (5.4) we set

$$
V_{n}^{(\omega)}:=\left\{v \in \ell^{2}\left(\mathbb{Z}^{d}\right) \mid(H(\omega)-\lambda) v=0 \text { and } \operatorname{supp} v \subseteq \Lambda_{n-R(n)}\right\}, \quad D_{n}^{(\omega)}:=\operatorname{dim} V_{n}^{(\omega)} .
$$

Note that $V_{n}^{(\omega)}$ consists of the elements $i_{\Lambda_{n}} v$, where $v \in \ell^{2}\left(\Lambda_{n}\right)$ satisfying $v \equiv 0$ on $\Lambda_{n} \backslash$ $\Lambda_{n-R(n)}$,

$$
\left(p_{\Lambda_{n}} H(\omega) i_{\Lambda_{n}}-\lambda\right) v=0 \quad \text { and } \quad \sum_{y \in \Lambda_{n-R(n)}}\left(H_{x, y}(\omega)-\lambda \delta_{x}(y)\right) v(y)=0
$$

for all $x \in \Lambda_{n}^{\mathrm{c}}$ with $x \stackrel{\omega}{\sim} \Lambda_{n-R(n)}$.

We consider the following difference

$$
\left|\rho_{n}^{(\omega)}(\{\lambda\})-\operatorname{Tr}\left(\chi_{\Lambda_{n}} E_{H(\omega)}\right)\right| \leq\left|\rho_{n}^{(\omega)}(\{\lambda\})-D_{n}^{(\omega)}\right|+\left|D_{n}^{(\omega)}-\operatorname{Tr}\left(\chi_{\Lambda_{n}} E_{H(\omega)}\right)\right|
$$

and treat the two summands on the right hand side separately. Let us estimate the first one. Consider therefore the sets

$$
U_{n}^{(\omega)}:=\left\{u \in \ell^{2}\left(\Lambda_{n}\right) \mid\left(p_{\Lambda_{n}} H(\omega) i_{\Lambda_{n}}-\lambda\right) u=0\right\}
$$

and

$$
U_{n, R}^{(\omega)}=\left\{u \in U_{n} \mid u \equiv 0 \text { on } \Lambda_{n} \backslash \Lambda_{n-R(n)}\right\} .
$$


Then clearly, $\rho_{n}^{(\omega)}(\{\lambda\})=\operatorname{dim}\left(U_{n}^{(\omega)}\right) \geq \operatorname{dim}\left(V_{n}^{(\omega)}\right)$ and

$$
\operatorname{dim}\left(U_{n, R}^{(\omega)}\right)-\operatorname{dim}\left(V_{n}^{(\omega)}\right) \leq\left|\left\{y \in \Lambda_{n}^{\mathrm{c}} \mid y \stackrel{\omega}{\sim} \Lambda_{n-R(n)}\right\}\right| \leq L^{(\omega)}\left(R(n), \Lambda_{n}\right)=L_{n}^{(\omega)},
$$

where we used the definition (5.3). The application of Lemma 5.3 gives

$$
\begin{aligned}
0 \leq \rho_{n}^{(\omega)}(\{\lambda\})-D_{n}^{(\omega)}=\operatorname{dim}\left(U_{n}^{(\omega)}\right)-\operatorname{dim}\left(V_{n}^{(\omega)}\right) & \leq \operatorname{dim}\left(U_{n}^{(\omega)}\right)-\operatorname{dim}\left(U_{n, R}^{(\omega)}\right)+L_{n}^{(\omega)} \\
& \leq\left|\partial^{R(n)} \Lambda_{n}\right|+L_{n}^{(\omega)}
\end{aligned}
$$

Now we estimate the second summand in (5.8). Therefore let $v_{1}, \ldots, v_{D_{n}^{(\omega)}}$ be an orthonormal basis (ONB) of $V_{n}^{(\omega)}$ and let $\tilde{v}_{i}, i \in I$ be an ONB of the orthogonal complement of $V_{n}^{(\omega)}$ in the space $\operatorname{Eig}(H(\omega), \lambda)$. Furthermore let $\bar{v}_{j}, j \in J$ be an $\mathrm{ONB}$ of $\operatorname{Eig}(H(\omega), \lambda)^{\perp}$. Then we have

$$
\begin{aligned}
\operatorname{Tr}\left(\chi_{\Lambda_{n}} E_{\omega}(\{\lambda\})\right) & =\sum_{i=1}^{D_{n}^{(\omega)}}\left\langle\chi_{\Lambda_{n}} E_{\omega}(\{\lambda\}) v_{i}, v_{i}\right\rangle+\sum_{i \in I}\left\langle\chi_{\Lambda_{n}} E_{\omega}(\{\lambda\}) \tilde{v}_{i}, \tilde{v}_{i}\right\rangle+\sum_{i \in J}\left\langle\chi_{\Lambda_{n}} E_{\omega}(\{\lambda\}) \bar{v}_{i}, \bar{v}_{i}\right\rangle \\
& =\sum_{i=1}^{D_{n}^{(\omega)}}\left\langle v_{i}, v_{i}\right\rangle+\sum_{i \in I}\left\langle\chi_{\Lambda_{n}} \tilde{v}_{i}, \chi_{\Lambda_{n}} \tilde{v}_{i}\right\rangle
\end{aligned}
$$

which gives $D_{n}^{(\omega)} \leq \operatorname{Tr}\left(\chi_{\Lambda_{n}} E_{\omega}(\{\lambda\})\right)$. Now let $u_{i}, i \in I$ be an ONB of

$$
\bar{U}_{n}^{(\omega)}:=\operatorname{ran}\left(\chi_{\Lambda_{n}} E_{\omega}(\{\lambda\})\right)
$$

and $\tilde{u}_{j}, j \in J$ be an ONB of $\left(\bar{U}_{n}^{(\omega)}\right)^{\perp}$. Then, using Cauchy Schwarz inequality, we obtain

$$
\left\langle\chi_{\Lambda_{n}} E_{\omega}(\{\lambda\}) u_{i}, u_{i}\right\rangle \leq\left\|\chi_{\Lambda_{n}} E_{\omega}(\{\lambda\}) u_{i}\right\|\left\|u_{i}\right\| \leq 1 \quad \text { and } \quad\left\langle\chi_{\Lambda_{n}} E_{\omega}(\{\lambda\}) \tilde{u}_{j}, \tilde{u}_{j}\right\rangle=0
$$

for all $i \in I$ and all $j \in J$. This gives

$$
D_{n}^{(\omega)} \leq \operatorname{Tr}\left(\chi_{\Lambda_{n}} E_{\omega}(\{\lambda\})\right)=\sum_{i \in I}\left\langle\chi_{\Lambda_{n}} E_{\omega}(\{\lambda\}) u_{i}, u_{i}\right\rangle+\sum_{j \in J}\left\langle\chi_{\Lambda_{n}} E_{\omega}(\{\lambda\}) \tilde{u}_{j}, \tilde{u}_{j}\right\rangle \leq \operatorname{dim}\left(\bar{U}_{n}^{(\omega)}\right) .
$$

where we used $\operatorname{dim}\left(\bar{U}_{n}\right)=|I|$. As before we denote by $\bar{U}_{n, R}^{(\omega)}$ the subset of $\bar{U}_{n}^{(\omega)}$ consisting of the elements which vanish outside of $\Lambda_{n-R}$. Therefore we have

$$
\bar{U}_{n, R}^{(\omega)}=\left\{\chi_{\Lambda_{n}} v \mid v \in \ell^{2}\left(\mathbb{Z}^{d}\right),(H(\omega)-\lambda) v=0, v \equiv 0 \text { on } \partial^{R(n)} \Lambda_{n}\right\} .
$$

In the next step we define a set $\bar{U}_{n, R}^{(\omega)} \supseteq \bar{U}_{n, R}^{(\omega)}$ by dropping conditions in (15.12), in the following way

$$
\begin{aligned}
\overline{\bar{U}}_{n, R}^{(\omega)} & :=\left\{\chi_{\Lambda_{n}} v \mid v \in \ell^{2}\left(\mathbb{Z}^{d}\right), \sum_{y \in \mathbb{Z}^{d}}\left(H_{x, y}(\omega)-\lambda \delta_{x}(y)\right) v(y)=0 \text { for all } x \in Z_{n}^{(\omega)}, v \equiv 0 \text { on } \partial^{R(n)} \Lambda_{n}\right\} \\
& =\left\{\chi_{\Lambda_{n}} v \mid v \in \ell^{2}\left(\mathbb{Z}^{d}\right), \sum_{y \in \Lambda_{n}}\left(H_{x, y}(\omega)-\lambda \delta_{x}(y)\right) v(y)=0 \text { for all } x \in Z_{n}^{(\omega)}, v \equiv 0 \text { on } \partial^{R(n)} \Lambda_{n}\right\},
\end{aligned}
$$


where

$$
Z_{n}^{(\omega)}=\Lambda_{n-R(n)} \backslash\left\{x \in \Lambda_{n-R(n)} \mid x \stackrel{\omega}{\sim} \Lambda_{n}^{\mathrm{c}}\right\} .
$$

Here we used that for all $x \in Z_{n}^{(\omega)}$ and $y \in \Lambda_{n}^{\mathrm{c}}$ we have $H_{x, y}(\omega)=0$.

Comparing this representation of $\overline{\bar{U}}_{n, R}^{(\omega)}$ with the representation $V_{n}^{(\omega)}$ in (5.7), we realize that they differ in at most $2 L_{n}^{(\omega)}+\left|\partial^{R(n)} \Lambda_{n}\right|$ conditions. As each of these conditions may change the dimension at most by one, we get

$$
\operatorname{dim}\left(\bar{U}_{n, R}^{(\omega)}\right) \leq \operatorname{dim}\left(\overline{\bar{U}}_{n, R}^{(\omega)}\right) \leq D_{n}^{(\omega)}+2 L_{n}^{(\omega)}+\left|\partial^{R(n)} \Lambda_{n}\right| .
$$

Applying (5.11), Lemma 5.3 and (5.13) gives

$$
\begin{aligned}
0 \leq \operatorname{Tr}\left(\chi_{\Lambda_{n}} E_{\omega}(\{\lambda\})\right)-D_{n}^{(\omega)} \leq \operatorname{dim}\left(\bar{U}_{n}^{(\omega)}\right)-D_{n}^{(\omega)} & \leq \operatorname{dim}\left(\bar{U}_{n, R}^{(\omega)}\right)-D_{n}^{(\omega)}+\left|\partial^{R(n)} \Lambda_{n}\right| \\
& \leq 2\left|\partial^{R(n)} \Lambda_{n}\right|+2 L_{n}^{(\omega)}
\end{aligned}
$$

In the last step we apply Lemma 5.2, then we combine the estimates for the two summands in (5.8) given in (5.10) and (5.14) and finally use part (b) of Lemma 5.4 to obtain

$$
\begin{aligned}
\lim _{n \rightarrow \infty} \frac{\rho_{n}^{(\omega)}(\{\lambda\})}{\left|\Lambda_{n}\right|}-\mathbb{E}\left(\left\langle E_{H(\omega)}(\{\lambda\}) \delta_{0}, \delta_{0}\right\rangle\right) & =\lim _{n \rightarrow \infty} \frac{\left|\rho_{n}^{(\omega)}(\{\lambda\})-\operatorname{Tr}\left(\chi_{\Lambda_{n}} E_{H(\omega)}\right)\right|}{\left|\Lambda_{n}\right|} \\
& \leq \lim _{n \rightarrow \infty} \frac{3\left|\partial^{R(n)} \Lambda_{n}\right|+3 L_{n}^{(\omega)}}{\left|\Lambda_{n}\right|} \\
& \leq 3 \lim _{n \rightarrow \infty}\left(\frac{\left|\partial^{R(n)} \Lambda_{n}\right|}{\left|\Lambda_{n}\right|}+\varepsilon(n)+\delta(n)\right)=0 .
\end{aligned}
$$

Here we used the definitions of $R(n), \varepsilon(n)$ and $\delta(n)$ in (5.4) and (5.6).

Remark 5.6. (a) Let us stress the fact that proof of Theorem 5.5 does not contain any probabilistic argument. We show the claimed convergence for any fixed choice of $\lambda \in \mathbb{R}$ and $\omega \in \tilde{\Omega}$, where $\tilde{\Omega}$ is a set given rather explicitly by Lemmas 5.2 and 5.4 .

(b) Furthermore the proof gives an explicit error-term on finite scales. To be precise we have for any $n \in \mathbb{N}, \lambda \in \mathbb{R}$ and $\omega \in \tilde{\Omega}$

$$
\left|\rho_{n}^{(\omega)}(\{\lambda\})-\operatorname{Tr}\left(\chi_{\Lambda_{n}} E_{H(\omega)}\right)\right| \leq 3\left|\partial^{R(n)} \Lambda_{n}\right|+3 L_{n}^{(\omega)}
$$

where $L_{n}^{(\omega)}=L^{(\omega)}\left(R(n), \Lambda_{n}\right)$ as in (5.9).

The following result is essentially standard and has been used in the present context already in [22]. It shows that weak convergence of measures plus convergence of the measures at each point implies uniform convergence.

Lemma 5.7. Let $\rho$ be a probability measure on $\mathbb{R}$ and let $\left(\rho_{n}\right)$ be as sequence of bounded measures on $\mathbb{R}$ which weakly converge to $\rho$ and fulfill

$$
\lim _{n \rightarrow \infty} \rho_{n}(\{\lambda\})=\rho(\{\lambda\})
$$

for all $\lambda \in \mathbb{R}$. Then the distribution functions $F_{n}: \mathbb{R} \rightarrow \mathbb{R}, F_{n}(\lambda):=\rho_{n}((-\infty, \lambda])$ converge with respect to supremum norm to the distribution function $F: \mathbb{R} \rightarrow \mathbb{R}, F(\lambda):=\rho((-\infty, \lambda])$. 
The proof of the main theorem, already stated in Section 2 is now basically a combination of the previous lemmas.

Proof of Theorem [2.1. Let $\rho, \rho_{n}^{(\omega)}: \mathcal{B}(\mathbb{R}) \rightarrow[0,1]$ be the measures associated to the distribution functions $F$ respectively $F_{n}^{\omega}$. Then obviously $\rho$ is a probability measure and the measures $\rho_{n}^{(\omega)}$ are bounded. As shown in Corollary 4.2 , there exists a set $\Omega_{1} \subseteq \Omega$ with $\mathbb{P}\left(\Omega_{1}\right)=1$ such that for all $\omega \in \Omega_{1}$ the measure $\rho$ is the weak limit of $\rho_{n}^{(\omega)}$. Furthermore we have by Theorem 5.5 a set $\Omega_{2} \subseteq \Omega$ with $\mathbb{P}\left(\Omega_{2}\right)=1$ such that for all $\omega \in \Omega_{2}$ and all $\lambda \in \mathbb{R}$ one has $\rho_{n}^{(\omega)}(\{\lambda\}) \rightarrow \rho(\{\lambda\})$. Therefore Lemma 5.7 yields the uniform convergence of the distribution functions for all $\omega \in \Omega_{1} \cap \Omega_{2}$.

\section{Acknowledgment}

The authors thank Christoph Schumacher for fruitful discussions.

\section{References}

[1] T. Antunović and I. Veselić. Equality of Lifshitz and van Hove exponents on amenable Cayley graphs. J. Math. Pures Appl., 92(4):342-362, 2009.

[2] T. Antunović and I. Veselić. Spectral asymptotics of percolation hamiltonians on amenable cayley graphs. In J. Janas, P. Kurasov, S. Naboko, A. Laptev, and G. Stolz, editors, Methods of Spectral Analysis in Mathematical Physics, volume 186 of Operator Theory: Advances and Applications, pages 1-29. Birkhäuser, Basel, 2009.

[3] S. Ayadi. Asymptotic properties of random matrices of long-range percolation model. Random Oper. Stoch. Equ., 17(4):295-1009, 2009.

[4] S. Ayadi. Semicircle law for random matrices of long-range percolation model. Random Oper. Stoch. Equ., 17(1):1-35, 2009.

[5] J. Bourgain and C. Kenig. On localization in the continuous Anderson-Bernoulli model in higher dimension. Invent. Math., 161:389-426, 2005.

[6] J. Bourgain and A. Klein. Bounds on the density of states for Schrödinger operators. Invent. Math., DOI 10.1007/s00222-012-0440-1, 2012.

[7] J. Chayes, L. Chayes, J. Franz, J. Sethna, and S. Trugman. On the density of states for the quantum percolation problem. J. Phys. A, 19(18):L1173-L1177, 1986.

[8] W. Craig and B. Simon. Log Hölder continuity of the integrated density of states for stochastic Jacobi matrices. Commun. Math. Phys., 90:207-218, 1983.

[9] W. Craig and B. Simon. Subharmonicity of the Lyaponov index. Duke Math. J., 50:551560, 1983.

[10] F. Delyon and B. Souillard. Remark on the continuity of the density of states of ergodic finite-difference operators. Commun. Math. Phys., 94:289-291, 1984. 
[11] J. Dodziuk, P. Linnell, V. Mathai, T. Schick, and S. Yates. Approximating $L^{2}$-invariants, and the Atiyah conjecture. Comm. Pure Appl. Math., 56(7):839-873, 2003.

[12] A. Figotin. Ergodic properties and essential self-adjointness of random matrix operators. In Operators in function spaces and problems in function theory, Collect. sci. Works, pages 13-27. Kiew, 1987.

[13] G. Keller. Equilibrium States in Ergodic Theory, volume 42 of London Mathematical Society Student Texts. Cambridge University Press, Cambridge, 1998.

[14] W. Kirsch. An invitation to random Schrödinger operators. In Random Schrödinger operators, volume 25 of Panoramas et synthèses, pages 1-119. Société Mathématique de France, 2008. with an appendix by Frédéric Klopp.

[15] W. Kirsch and P. Müller. Spectral properties of the Laplacian on bond-percolation graphs. Math. Zeit., 252(4):899-916, 2006.

[16] S. Klassert, D. Lenz, and P. Stollmann. Discontinuities of the integrated density of states for random operators on Delone sets. Comm. Math. Phys., 241(2-3):235-243, 2003.

[17] A. Klenke. Probability Theory - A Comprehensive Course. Springer Verlag, London, 2008.

[18] D. Lenz, P. Müller, and I. Veselić. Uniform existence of the integrated density of states for models on $\mathbb{Z}^{d}$. Positivity, 12(4):571-589, 2008.

[19] D. Lenz, F. Schwarzenberger, and I. Veselić. A Banach space-valued ergodic theorem and the uniform approximation of the integrated density of states. Geometriae Dedicata, 150(1):1-34, 2010.

[20] D. Lenz and P. Stollmann. Aperiodic order and quasicrystals: spectral properties. Ann. Henri Poincaré, 4(suppl. 2):S933-S942, 2003.

[21] D. Lenz and P. Stollmann. An ergodic theorem for Delone dynamical systems and existence of the density of states. J. Anal. Math., 97:1-23, 2006.

[22] D. Lenz and I. Veselić. Hamiltonians on discrete structures: jumps of the integrated density of states and uniform convergence. Math. Z., 263(4):813-835, 2009.

[23] E. Lindenstrauss. Pointwise theorems for amenable groups. Invent. Math., 146(2):259295, 2001.

[24] W. Lück. Approximating $L^{2}$-invariants by their finite-dimensional analogues. Geom. Funct. Anal., 4(4):455-481, 1994.

[25] V. Mathai, T. Schick, and S. Yates. Approximating spectral invariants of Harper operators on graphs. II. Proc. Amer. Math. Soc., 131(6):1917-1923 (electronic), 2003.

[26] V. Mathai and S. Yates. Approximating spectral invariants of Harper operators on graphs. J. Funct. Anal., 188(1):111-136, 2002. arXiv.org/math.FA/0006138.

[27] P. Müller and P. Stollmann. Spectral asymptotics of the Laplacian on supercritical bond-percolation graphs. J. Funct. Anal., 252:233-246, 2007. 
[28] L. Pastur and A. Figotin. Spectra of Random and Almost-Periodic Operators. Springer Verlag, Berlin, 1992.

[29] F. Pogorzelski and F. Schwarzenberger. A Banach space-valued ergodic theorem for amenable groups and applications. arXiv:math/1205.3649, 2012.

[30] F. Schwarzenberger. Uniform approximation of the integrated density of states for longrange percolation Hamiltonians. J. Stat. Phys., 146(6):1156-1183, 2012.

[31] F. Schwarzenberger. The integrated density of states for operators on groups. $\mathrm{PhD}$ thesis, Technische Universität Chemnitz, 2013. submitted.

[32] I. Veselić. Spectral analysis of percolation Hamiltonians. Math. Ann., 331(4):841-865, 2005.

[33] I. Veselić. Existence and Regularity Properties of the Integrated Density of States of Random Schrödinger Operators, volume 1917 of Lecture Notes in Mathematics. Springer, 2008.

[34] F. Wegner. Bounds on the DOS in disordered systems. Z. Phys. B, 44(1-2):9-15, 1981. 